\title{
Magnetic Resonance Imaging Manifestations of Bilateral Cerebral Amyloid Angiopathy-Related Inflammation with Seizures
}

Yasunobu Nosaki*, Ken Ohyama, Maki Watanabe, Takamasa Yokoi, Katsushige Iwai, and Kenji Mokuno

Department of Neurology, Toyohashi Municipal Hospital, Toyohashi, Japan

\begin{abstract}
Cerebral Amyloid Angiopathy-related inflammation (CAA-ri) is a distinct subtype of CAA characterized by rapidly progressive cognitive decline, focal deficits, seizures, and headache. It is characterized by vasculitis associated with cerebral amyloid deposition. Differential diagnosis may be challenging due to overlapping symptoms and lack of clear imaging criteria, although CCA-ri is usually responsive to immunosuppressive therapy. We describe an 81-year-old female diagnosed with CAA-ri by Magnetic Resonance Imaging (MRI). Fluid-Attenuated Inversion Recovery (FLAIR) imaging revealed hyperintense lesions in the bilateral temporal and occipital areas, and $\mathrm{T} 2^{*}$-weighted gradient echo (T2*-GRE) imaging demonstrated multiple cortical and subcortical hypointensities in the same areas. Furthermore, the findings of T2*-GRE were confirmed by Susceptibility-Weighted Imaging (SWI). In cases of encephalopathy, CAA-ri should be considered in the differential diagnosis, and the presence of microbleeds should be examined using T2*-GRE and (or) SWI MRI.
\end{abstract}

Keywords: Amyloid angiopathy; Cerebral Amyloid Angiopathyrelated inflammation (CAA-ri); MRI; Susceptibility-Weighted Imaging (SWI)

\section{Case Report}

An 81-year-old female was admitted with generalized seizures.
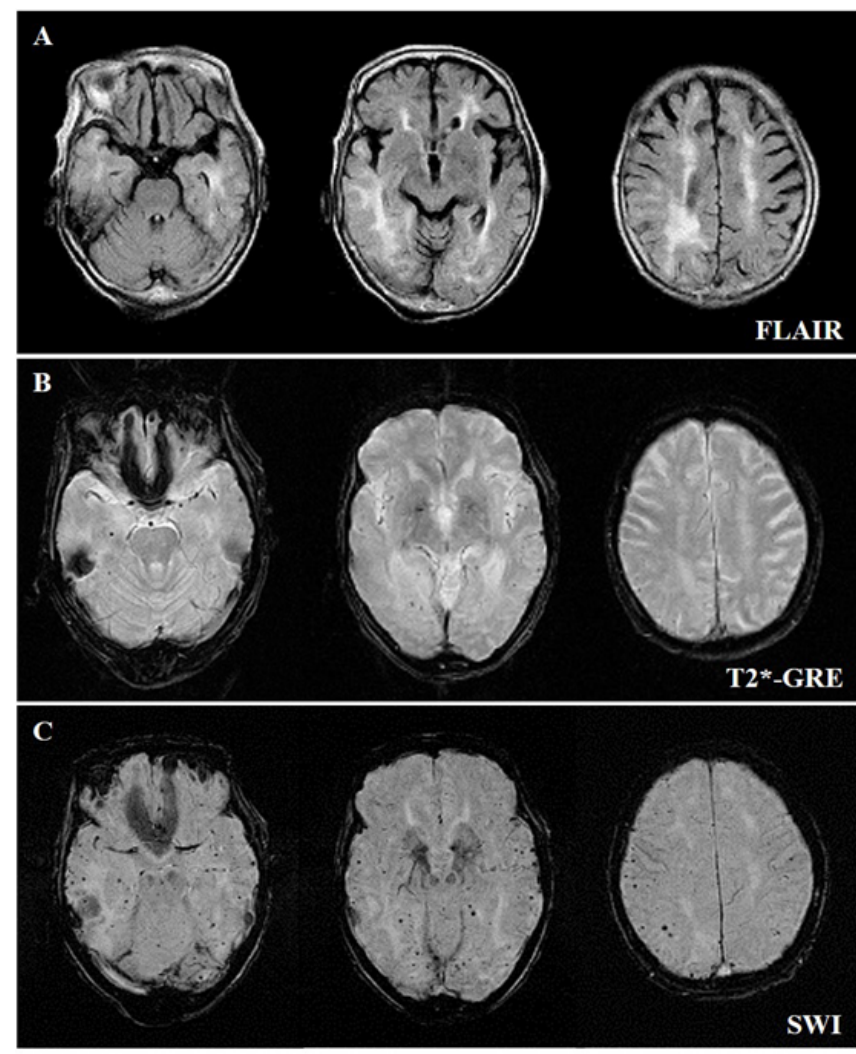

Figure 1: Brain Magnetic Resonance Imaging (MRI) revealed hyperintense lesions in the bilateral temporal and occipital areas on Fluid-Attenuated Inversion Recovery (FLAIR) sequences.
After the seizures resolved, severe cognitive dysfunction was observed. Laboratory examinations, including a cerebrospinal fluid study, detected no abnormalities. The electroencephalogram exhibited frequent epileptic discharges in the right mid-temporal area. Brain Magnetic Resonance Imaging (MRI) revealed hyperintense lesions in the bilateral temporal and occipital areas on Fluid-Attenuated Inversion Recovery (FLAIR) sequences (Figure 1A). T2*-weighted gradient echo (T2*-GRE) imaging demonstrated multiple cortical and subcortical hypointensities in the same areas (Figure 1B). Moreover, T2*-GRE findings were confirmed by Susceptibility-Weighted Imaging (SWI) (Figure 1C). Although brain biopsy was not performed in the patient due to clinical deterioration, we diagnosed her with Cerebral Amyloid Angiopathy-related inflammation (CAA-ri) based on diagnostic criteria (1), and initiated treatment with antiepileptic drugs and corticosteroids. The patient became seizure-free and MRI findings revealed slight improvement of the bilateral temporal lobe lesions; however, severe cognitive dysfunction remained.

Cerebral Amyloid Angiopathy-related inflammation (CAA-ri) is characterized by vasculitis associated with cerebral amyloid deposition, which induces rapidly progressive cognitive decline, focal deficits, seizures, and headache [1-2]. The MRI manifestations of CAA-ri include patchy or confluent hyperintensity on T2-weighted or FLAIR images, and the presence of multiple cortical and subcortical hemorrhages [1]. Although there are usually asymmetric inflammatory lesions, multifocal hyperintensities or bilateral changes are found in $25 \%-40 \%$

*Corresponding author: Yasunobu Nosaki, Department of Neurology, Toyohash Municipal Hospital, Japan, Tel: +81-532-33-6111; Fax: +81-532-33-6177, E-mail: nozaki-yasunobu@toyohashi-mh.jp

Received December 07, 2018; Accepted December 26, 2018; Published December 31, 2018

Citation: Nosaki Y, Ohyama K, Watanabe M, Yokoi T, Iwai K, et al. (2018) Magnetic Resonance Imaging Manifestations of Bilateral Cerebral Amyloid Angiopathy-Related Inflammation with Seizures. J Neurol Neurophysiol 9: 476. doi:10.4172/2155-9562.1000476

Copyright: (C) 2018 Nosaki Y, et al. This is an open-access article distributed under the terms of the Creative Commons Attribution License, which permits unrestricted use, distribution, and reproduction in any medium, provided the original author and source are credited. 
Citation: Nosaki Y, Ohyama K, Watanabe M, Yokoi T, Iwai K, et al. (2018) Magnetic Resonance Imaging Manifestations of Bilateral Cerebral Amyloid Angiopathy-Related Inflammation with Seizures. J Neurol Neurophysiol 9: 476. doi:10.4172/2155-9562.1000476

of cases [2,3]. Differential diagnosis may include infections, sarcoidosis, acute disseminated encephalomyelitis, lymphoma, and brain tumors. CAA-ri may be distinguished by responsivity to immunosuppressive therapy.

\section{Conclusion}

Therefore, in cases of encephalopathy presenting with cognitive decline or seizure, CAA-ri should be considered and the presence of microbleeds should be examined by T2*-GRE or SWI MRI [1,2].

\section{References}

1. Chung KK, Anderson NE, Hutchinson D, Synek B, Barber PA (2011) Cerebral amyloid angiopathy related inflammation: Three case reports and a review. $J$ Neurol Neurosurg Psychiatry 82: 20-26.

2. Ronsin S, Deiana G, Geraldo AF, Durand-Dubief F, Thomas-Maisonneuve L, et al. (2016) Pseudotumoral presentation of cerebral amyloid angiopathy-related inflammation. Neurology 86: 912-919.

3. Corovic A, Kelly S, Markus HS (2018) Cerebral amyloid angiopathy associated with inflammation: A systematic review of clinical and imaging features and outcome. Int J Stroke 13: 257-267. 